\title{
Human fungal pathogens: Why should we learn?
}

\author{
Jeong-Yoon Kim \\ Department of Microbiology and Molecular Biology, College of Bioscience \\ and Biotechnology, Chungnam National University, Daejeon 34134, \\ Republic of Korea
}

Human fungal pathogens that cause invasive infections are hidden killers, taking lives of one and a half million people every year. However, research progress in this field has not been rapid enough to effectively prevent or treat life-threatening fungal diseases. To update recent research progress and promote more active research in the field of human fungal pathogens, eleven review articles concerning the virulence mechanisms and host interactions of four major human fungal pathogens-Candida albicans, Cryptococcus neoformans, Aspergillus fumigatus, and Histoplasma capsulatum-are presented in this special issue.

Keywords: human fungal pathogens, invasive infection, Candida albicans, Cryptococcus neoformans, Aspergillus fumigatus, Histoplasma capsulatum

\section{Introduction}

Fungi can infect humans and cause various diseases from superficial infections of the skin and mucosal surfaces to invasive infections of internal organs. While superficial infections are common and generally easy to cure, invasive infections have a much lower incidence rate than superficial infections and cause life-threatening diseases, particularly in immunocompromised patients with HIV/AIDS or autoimmune diseases and in those undergoing anti-cancer chemotherapy or organ transplantation. Paradoxically, modern medicine has greatly increased the number of immunosuppressed patients who are at high risk for invasive infections. Human pathogenic fungi causing invasive infections kill about one and a half million people every year, giving them the name hidden killers (Brown et al., 2012). Nonetheless, research on human fungal pathogens has not progressed as much as research on other microbial pathogens, and the slow progress in the field has hampered the development of novel antifungal drugs as well as techniques for diagnosing invasive fungal diseases.

Among the estimated 3.5-5.1 million fungal species (O’Brien

${ }^{\star}$ For correspondence. E-mail: jykim@cnu.ac.kr

Copyright (C) 2016, The Microbiological Society of Korea et al., 2005), only several hundred species are associated with human fungal diseases (Köhler et al., 2014). Just a small number of species among the human pathogenic fungi cause the most common invasive infections in immunocompromised individuals. This special issue provides eleven review articles concerning four human fungal pathogens that cause invasive infections - C. albicans, C. neoformans, A. fumigatus, and $H$. capsulatum. C. albicans is a commensal fungus which exists as a normal microflora in the human body but becomes pathogenic if the host immune defense system is weakened. C. neoformans, A. fumigatus, and $H$. capsulatum are environmental pathogens, and spores or yeast form cells that are accidentally inhaled cause invasive infections in immunocompromised patients (Reedy et al., 2007).

The objectives of this special issue are to compile the already existing findings about human fungal pathogens and give directions for future research in the field. Contributors provide a comprehensive review on a range of research subjects: essential concepts of fungal pathogenesis, molecular mechanisms of morphogenesis and virulence, interactions between pathogenic fungi and host cells, involvement of cellular structures in virulence, developmental regulators of pathogenic fungi, and the emergence of drug-resistance strains. This special issue also includes reviews about the host immune system and mechanisms to evade it.

\section{Candida albicans}

C. albicans is a harmless constituent of the human microbiota. However, when the balanced interaction between $C$. albicans and host cells is disrupted, C. albicans becomes a pathogen overgrowing on skin and mucosal surfaces and/or invading the host tissue, disseminating into the bloodstream, and colonizing in solid organs. Four articles provide fascinating reviews on the interaction between $C$. albicans and the environment, with an emphasis on the host defense system and bacterial microbiota, hyphal morphogenesis, roles of special membrane architectures in pathogenesis, and the microevolution of antifungal drug resistance.

Höfs et al. (2016) discuss how the host interaction is maintained and disturbed, the factors that can be attributed to the dynamics of the interaction, and the ways in which bacteria in the microbiota interact with $C$. albicans to exert positive or negative effects on the virulence potential of $C$. albicans. Furthermore, they describe the molecular basis of the host defense strategies, innate and adaptive immune responses, C. albicans strategies of evasion from the host defense sys- 
tem, shielding of pathogen-associated molecular patterns, and white-opaque morphological switching. The authors end this review with an emphasis on the importance of the interaction between $C$. albicans and the bacterial microbiota in a C. albicans infection.

Since the yeast-hypha transition is a critical virulence factor of C. albicans, great efforts have been made for decades to find the key factors and elucidate the mechanisms that drive the morphological changes. $\mathrm{Hgcl}$ is hyphal-specific-G1 cyclin 1, which is not involved in the cell cycle but constitutively expressed during hyphal growth. Hgcl was discovered to be a key regulator of a C. albicans morphogenesis pathway that is activated through the CAMP-PKA pathway (Zheng et al., 2004). In this review, Wang (2016) brings together the progresses that have been made to elucidate the roles and molecular functions of $\mathrm{Hgcl}$ in multiple cellular processes required for C. albicans hyphal development. $\mathrm{Hgcl}$ is associated with diverse cellular machines responsible for different aspects of hyphal growth, such as cell polarity, membrane trafficking, and cell separation. Wang suggests that to fully understand the hyphal development of C. albicans, it is necessary to study how Hgc1-Cdc28 and its downstream effectors cooperate with other morphology regulators.

The plasma membrane and the ergosterol synthesis pathway are the main targets of commonly used antifungal drugs, the polyenes and the azoles, respectively. Douglas and Konopka (2016) review the composition and architecture of the distinct domains of the plasma membrane, which are the barriers to diffusion or scaffolds, such as the membrane compartment of Can1 (MCC)/eisosome, septins, and contact sites with the endoplasmic reticulum, or mediate dynamic processes, including protein secretion, endocytosis, and polarized growth. They present evidence that the components of MCC/ eisosomes, Sur7, Nce102, and FLP quinone reductases, are required for $C$. albicans virulence, and that septins play a special role in C. albicans hyphal morphogenesis. This indicates that further investigation of the distinct architectures of the plasma membrane will provide important insights about the roles of the plasma membrane in fungal pathogenesis. The authors also suggest that a better understanding of the structure, composition, and pathogenic relevance of fungal membranes will provide valuable information for developing new therapeutic drugs against C. albicans.

C. albicans acquires antifungal drug resistance due to mutations in genes whose functions are to diminish toxic byproducts, to alter the binding site to a drug or increase the amount of target enzymes, or to pump the drug out of the cell. In this review, Morschhäuser (2016) provides evidence of the molecular mechanisms by which fluconazole-resistant strains can be generated from an original clonal, drug-susceptible population. He also discusses fluconazole-driven genomic rearrangement events that can result in homozygosity for resistance mutations. Since the genomic rearrangement is often accompanied by MTL homozygosity (MTLa/a or $M T L \alpha / \alpha$ ), which is the mating-competent form (Miller and Johnson, 2002), fluconazole-resistant C. albicans strains can evolve into highly resistant strains through sexual recombination. This is an intriguing hypothesis that needs to be experimentally proven. Morschhäuser also suggests that it would be valuable to study how the fitness costs of drug re- sistance are overcome in many fluconazole-resistant clinical isolates.

\section{Cryptococcus neoformans}

C. neoformans is a basidiomycete fungal human pathogen which causes life-threatening fungal pneumonia and meningitis in immunocompromised patients. Another related species, Cryptococcus gattii, infects immunocompetent individuals in tropical areas; however, there is evidence that $C$. neoformans also infects immunocompetent hosts (Panackal et al., 2015). C. neoformans is unique among human fungal pathogens as it is encapsulated by polysaccharides. Because the capsule and melanin formation, virulence-associated enzymes, and signaling pathways for pathogenicity have been recently reviewed elsewhere (O'Meara and Alspaugh, 2012; Bahn and Jung, 2013; Almeida et al., 2015; Choi et al., 2015), this special issue presents two reviews on the latest findings of host immune responses to C. neoformans and our current understanding of lipid modification and its role in $C$. neoformans virulence.

The first review by Hole and Wormley (2016) focuses on innate host defenses against $C$. neoformans. Although adaptive cell-mediated immunity (CMI) is critical for eradicating the infecting $C$. neoformans, adaptive CMI does not function properly in most infected patients because they are immunosuppressed due to organ transplantation or other immune diseases. Thus, to develop effective therapies to help these patients overcome the cryptococcosis disease, an understanding of how the innate immune system combats $C$. neoformans is a prerequisite. Hole and Wormley describe the molecular mechanisms for innate immunity and the roles of phagocytic cells, such as macrophage, dendritic cells, and natural killer cells in phagocyting and killing C. neoformans. This article delivers the message that modulation of natural killer cells, macrophages, and dendritic cells to be more aggressive against C. neoformans should be considered as a therapeutic strategy for cryptococcosis.

Covalent lipid modification of proteins changes the physical and chemical properties of proteins for specific purposes and is thus regarded as an important regulatory entity in diverse cellular events. Until now, about 10 lipid modifications on different amino acid residues are known (Hentschel et al., 2015). Santiago-Tirado and Doering (2016) review enzymes, their substrates, and the processes involved in the lipid modification of proteins, such as $\mathrm{N}$-myristoylation, prenylation, S-acylation, and GPI-attachment. They further discuss the essential roles of lipid modification of proteins on the viability and virulence of $C$. neoformans. Although the core mechanisms and consequences of lipid modifications are common among eukaryotic cells, there are molecular differences in C. neoformans from the host cell. The unique features of $C$. neoformans in the lipid modification processes include enzymes, such as myristoyl-CoA:protein N-myristoyltransferase, inositol transferase, farnesyl transferase, and protein S-acyltransferase. The authors suggest that these enzymes may be promising antifungal targets. 


\section{Aspergillus fumigatus}

The airborne asexual spores, called conidia, of A. fumigatus are inhaled by humans but effectively cleared by the innate immune system in healthy individuals. However, the spores evade the defense system in immunocompromised individuals and cause the life-threatening invasive aspergillosis. No single characteristic determines the pathogenicity of $A$. fumigatus; its ability to adapt to high temperatures, oxidative stress, nutrient limitations, and hypoxic conditions, as well as to synthesize secondary metabolites and secrete enzymes for nutrient uptake all contribute to the survival of $A$. fumigatus inside the human body. This special issue contains four reviews that discuss a variety of aspects associated with the pathogenicity of $A$. fumigatus: regulation of conidia production, understanding of cell wall biosynthesis, the relevance of the stress responses to virulence, and oxypilin and host-pathogen interactions.

Conidia are required for the reproduction, survival, and infectivity of A. fumigatus. Park and Yu (2016) provide a review of the key regulators of conidiation in A. fumigatus. They describe the functions and molecular mechanisms of three key elements, BrlA, AbaA, and WetA, that govern conidiation-specific gene expression, upstream activators and repressors of BrlA, and the velvet family proteins which are fungal-specific transcription factors. Park and Yu also discuss the involvement of heterotrimeric $G$ protein signaling pathways, the $\mathrm{Ca}^{2+}$-Calcineurin pathway, and the Ras-mediated signaling pathway in the asexual development and hyphal growth of A. fumigatus. They suggest that more comprehensive and detailed studies about the regulatory mechanisms of conidiation need to be carried out in order to develop novel approaches to control the pathogenicity of $A$. fumigatus.

The next review by Lee and Sheppard (2016) introduces recent advances in the understanding of the A. fumigatus cell wall. It is generally accepted that the fungal cell wall is an attractive target for antifungal drugs because it is essential for growth and morphogenesis and absent in human cells. Furthermore, components of the cell wall serve as pathogenassociated molecular patterns that are recognized by cells of the innate immunity system. Thus, understanding the structure and biosynthesis of the fungal cell wall is of utmost importance in the research field of fungal pathogens. In this review, Lee and Sheppard compared the cell walls of conidia and hyphae. They describe the function and synthesis of each cell wall polysaccharide and discuss the regulatory mechanisms for cell wall remodeling during cell wall stress or damage. More studies are expected to further unravel the cell wall dynamics of A. fumigatus and other pathogens during infection. Consequently, new classes of antifungals that target the cell wall will be lined up with echinocandins, the antifungals that inhibit $\beta-1,3$ glucan synthase activity.

Once they escape from the alveolar macrophages in the lung, conidia of A. fumigatus germinate and adapt to the hostile host environment. Brown and Goldman (2016) describe the stress conditions A. fumigatus should overcome to survive in the host. They also review the molecular mechanisms of how A. fumigatus operates signaling pathways to cope with nutrient limitations and imbalance, cell wall stress, oxidative stress, an alkaline $\mathrm{pH}$, and high temperatures. They also mention that the highly conserved fungal stress response pathways satisfy the criteria for potential antifungal drug targets. Accordingly, they suggest that therapies that utilize inhibitors of the $\mathrm{Ca}^{2+}$-calcineurin signaling pathway, Hsp90, or oxidative stress response pathway in combination with current antifungals should increase the efficacy or decrease the dosage of the antifungals.

Oxylipins are bioactive lipids produced by the oxygenation of polyunsaturated fatty acids. It is generally acknowledged that oxylipins are involved in fungal developmental program as well as environmental responses. In this review, Fischer and Keller (2016) summarize a variety of oxylipins produced by lipooxygenases, cyclooxygenases, and monooxygenases in fungi. They also update our knowledge on the roles of oxylipins in fungi and plant or fungi and mammal interactions. Fungal oxylipins can modulate host oxygenase expression. In addition, fungal oxylipins can be modified by mammalian cells and utilized as potent immune system-modulating oxylipins. This indicates that oxylipins may play an important role in host-pathogen interactions. Future research efforts will be made to identify oxylipins profiles from diverse fungal species and unravel the specific roles of oxylipins in fungihost interactions.

\section{Histoplasma capsulatum}

$H$. capsulatum is a thermally dimorphic fungus that grows in a hyphal form in the environment but exists as a budding yeast in mammalian hosts. H. capsulatum causes Histoplasma, which has similar clinical and pathogenic features of tuberculosis. Woods (2016) revisits the original description and phylogenetic classification, ecology, dimorphic characteristics, and infection events of $H$. capsulatum. He also introduces the detailed mechanisms that facilitate H. capsulatum pathogenesis. Woods describes the involvement of $\alpha$-1,3-glucan in shielding $\beta$-1,3-glucan, an important pathogen associated molecular pattern. The functional necessity in the pathogenesis of $H$. capsulatum oxidative resistance associated with extracellular superoxide dismutase and catalases are also discussed. Further, he reviews how H. capsulatum acquires nutrients, especially iron, from the host, and how Histoplasmaspecific genes, such as $C B P 1$ and YPS3, are involved in the virulence of $H$. capsulatum. Thus, this review definitely adds to our understanding of the basic biology of $H$. capsulatum and the important features responsible for $H$. capsulatum pathogenesis.

Finally, Journal of Microbiology is grateful to the authors for sharing their knowledge with our readers. The reviews in this special issue are expected to be essential references for pathogenic fungal biologists who wish to understand and develop strategies to control human fungal pathogens that cause invasive infections. The research field of human fungal pathogens will continue to expand, and important findings in the near future will lead to find creative ways for fighting against fatal fungal infections. 


\section{Acknowledgements}

I would like to thank the National Research Foundation of Korea (grant 2013R1A2A2A01014664) and Chungnam National University for financial support for this work.

\section{References}

Almeida, F., Wolf, J.M., and Casadevall, A. 2015. Virulence-associated enzymes of Cryptococcus neoformans. Eukaryot. Cell 14, 1173-1185.

Bahn, Y.S. and Jung, K.W. 2013. Stress signaling pathways for the pathogenicity of Cryptococcus. Eukaryot. Cell 12, 1564-1577.

Brown, G.D., Denning, D.W., Gow, N.A.R., Levits, S.M., Netea, M.G., and White, T.C. 2012. Hidden killers: Human fungal infections. Sci. Transl. Med. 4, 1-9.

Brown, N.A. and Goldman, G.H. 2016. The contribution of Aspergillus fumigatus stress responses to virulence and antifungal resistance. J. Microbiol. 54, 243-253.

Choi, J., Jung, W.H., and Kronstad, J.W. 2015. The cAMP/protein kinase A signaling pathway in pathogenic basidiomycete fungi: Connections with iron homeostasis. J. Microbiol. 53, 579-587.

Douglas, L.M. and Konopka, J.B. 2016. Plasma membrane organization promotes virulence of the human fungal pathogen Candida albicans. J. Microbiol. 54, 178-191.

Fischer, G.J. and Keller, N.P. 2016. Production of cross-kingdom oxylipins by pathogenic fungi: An update on their role in development and pathogenicity. J. Microbiol. 54, 254-264.

Hentschel, A., Zahedi, R.P., and Ahrends, R. 2015. Protein lipid modifications - more than a just a greasy ballast. Proteomics DOI: 10.1002/pmic.201500353.

Höfs, S., Mogavero, S., and Hube, B. 2016. Interaction of Candida albicans with host cells: virulence factors, host defence, escape strategies, and the microbiota. J. Microbiol. 54, 149-169.

Hole, C. and Wormley, F.L. 2016. Innate host defense against Crytococcus neoformans. J. Microbiol. 54, 202-211.

Köhler, J.R., Casadevall, A.C., and Perfect, J. 2014. The spectrum of fungi that infects human. Cold Spring Harb. Perspect. Med. 5, a019723.

Lee, M.J. and Sheppard, D.C. 2016. Recent advances in the understanding of the Aspergillus fumigatus cell wall. J. Microbiol. 54, 232-242.

Miller, M.G. and Johnson, A.D. 2002. White-opaque switching in Candida albicans is controlled by mating-type locus homeodomain proteins and allows efficient mating. Cell 110, 293-302.

Morschhäuser, J. 2016. The development of fluconazole resistance in Candida albicans - an example of microevolution of a fungal pathogen. J. Microbiol. 54, 192-201.

O’Brien, H.E., Parrent, J.L., Jackson, J.A., Moncalvo, J.M., and Vilgalys, R. 2005. Fungal community analysis by large-scale sequencing of environmental samples. Appl. Environ. Microbiol. 71, 55445550.

O'Meara, T.R. and Alspaugh, J.A. 2012. The Cryptococcus neoformans capsule: a sword and a shield. Clin. Microbiol. Rev. 25, 387-408.

Panackal, A.A., Wuest, S.C., Lin, Y.C., Wu, T., Zhang, N., Kosa, P., Komori, M., Blake, A., Browne, S.K., Rosen, L.B., et al. 2015. Paradoxical immune responses in non-HIV Cryptococcal meningitis. PLoS Pathog. 11, E1004884.

Park, H.S. and Yu, J.H. 2016. Developmental regulators in Aspergillus fumigatus. J. Microbiol. 54, 223-231.

Reedy, J.L., Bastidas, R.J., and Heitman, J. 2007. The virulence of human pathogenic fungi: Notes from the South of France. Cell Host Microbe 2, 77-83.

Santiago-Tirado, F.H. and Doering, T.L. 2016. All about that fat: Lipid modification of proteins in Cryptococcus neoformans. J. Microbiol. 54, 212-222.

Wang, Y. 2016. Hgc1-Cdc28-how much does a single protein kinase do in the regulation of hyphal development in Candida albicans. J. Microbiol. 54, 170-177.

Woods, J.P. 2016. Revisiting old friends: Developments in understanding Histoplasma capsulatum pathogenesis. J. Microbiol. 54, 265-276.

Zheng, X., Wang, Y., and Wang, Y. 2004. Hgc1, a novel hypha-specific G1 cyclin-related protein regulates Candida albicans hyphal morphogenesis. EMBO J. 23, 1845-1856. 